\title{
Poincaré-covariant quark model of electroweak light mesons decays
}

\author{
Viktor Andreev ${ }^{1, *}$ and Vadzim Haurysh ${ }^{2, * *}$ \\ ${ }^{1}$ Gomel State University named after F. Skorina, Gomel, Belarus \\ ${ }^{2}$ Sukhoi State Technical University of Gomel, Gomel, Belarus
}

\begin{abstract}
The procedure for obtaining the radiative decay constants of pseudoscalar and vector unflavored mesons in point-form of Poincaré-invariant quantum mechanics is presented. In the course of the work the authors determine the remaining parameters from $V \rightarrow P \gamma$ and $P \rightarrow V \gamma$ experimental decay value, using the obtained earlier parameters of the model for light $(u, d$ and $s)$ quarks. As a result, the mixing angles for pseudoscalar and vector meson sector were obtained taking into account gluonium content for $\eta-\eta^{\prime}$ meson sector.
\end{abstract}

\section{Introduction}

The problem of hadron decay descriptions, as a bound quark system, is an important task of high energy physics and theoretical physics. In view of several well-known problems of quantum electrodynamics there is a number of alternative models and approaches; nevertheless, any of these approaches requires further modifications and reviews, since the mechanics of the bound quark interaction has not been completely studied.

The research in this area has become particularly important nowadays because of the appearance of modern experimental data on lepton decays of light pseudoscalar and vector mesons, as well as for heavy lepton decays (see [1]). Due to these ones and more recent experimental research works, a series of papers has appeared which is dedicated to the calculations of various form factors of hadrons and other observables from these decays.

Making reference to the decays, which include hadron in the final state, processes with emitting $\gamma$-quantum $V(P) \rightarrow P(V) \ell^{+} \ell^{-}$and $V(P) \rightarrow P(V) \gamma$ are the most convenient for developing the theory of inner quark dynamics: it has been found out, that such decays have the simplest decay mechanism and allow one to find a number of phenomenological characteristics of hadrons, such as magnetic moments, different hadron form factors, mixing angles of quark and gluons etc. As a consequence, these processes are convenient for testing grounds of any theory to extend the picture of quark dynamics more completely than in the case of purely hadronic decays. The research of these decays also makes it possible not only to obtain the behaviour of transition form factors of mesons, but estimate the mixing angles for vector and pseudoscalar meson sectors: this problem is particularly important because of the advent of the latest experimental data on the decays of pseudoscalar $\eta$ and $\eta^{\prime}$-mesons $[2,3]$ there are known cases of analysis data with an additional mixing angle for gluonium content.

\footnotetext{
*e-mail: vik.andreev@ rambler.ru

**e-mail: mez0n@inbox.ru
} 
Despite the fact, that there is a number of papers dedicated to such kind of calculations in instant and front-form of Poincaré-invariant quantum mechanics (PiQM), the point-form of $\mathrm{PiQM}$, for several difficulties, is rarely used for these purposes. Therefore, further development of this form of dynamics is an important problem of hadron physics.

This article is organized as follows: section 1 briefly discusses the scheme of model parameters obtaining, section 2 demonstrates the procedure of integral representations calculation for $V(P) \rightarrow P(V) \gamma^{*}$ decay constant in PiQM with the followed obtaining the numerical estimation of quark magnetic moments. As a result of the work, sections 3 and 4 discuss and obtain mixing angles for unflavored mesons and remaining parameters of the model with following comparison model calculations with the experimental data.

\section{Parameters of the model, based on point-form of PiQM}

Here we introduce the basic parameters, obtained earlier in [4, 5]. Let us briefly discuss the basic steps of scheme calculations: using relation for the state vector of meson with momentum $\mathbf{Q}$, angular momentum $J$ and mass $M$ in point-form of PiQM

$$
\begin{aligned}
& |\mathbf{Q}, J \mu, M\rangle=\sum_{\lambda_{1}, \lambda_{2}} \sum_{v_{1}, \nu_{2}} \int \mathrm{d} \mathbf{k} \sqrt{\frac{\omega_{m_{q}}\left(\mathrm{p}_{1}\right) \omega_{m_{\bar{Q}}}\left(\mathrm{p}_{2}\right) M_{0}(\mathrm{k})}{\omega_{m_{q}}(\mathrm{k}) \omega_{m_{\bar{Q}}}(\mathrm{k}) \omega_{M_{0}}\left(\mathrm{P}_{12}\right)}} \Phi_{\ell S}^{J}\left(\mathrm{k}, \beta_{q \bar{Q}}^{I}\right) \times
\end{aligned}
$$

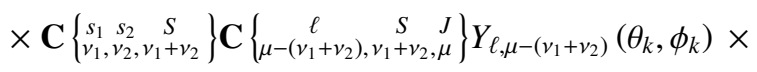

$$
\begin{aligned}
& \times D_{\lambda_{1}, v_{1}}^{1 / 2}\left(\mathbf{n}_{W_{1}}\right) D_{\lambda_{2}, v_{2}}^{1 / 2}\left(\mathbf{n}_{W_{2}}\right)\left|\mathbf{p}_{\mathbf{1}}, \lambda_{1}, \mathbf{p}_{2}, \lambda_{2}\right\rangle,
\end{aligned}
$$

one can obtain an integral representation of pseudoscalar, vector mesons $f_{P, V}\left(q, \bar{Q}, \beta_{q \bar{Q}}^{P, V}\right)$ as well as pseudoscalar density constant $g\left(q, \bar{Q}, \beta_{q \bar{Q}}^{P}\right)$ [5]. After some calculations with an oscillator wave function for pseudoscalar $(I=P, \ell=0)$ and vector mesons $(I=V, \ell=0)$

$$
\Phi\left(\mathrm{k}, \beta_{q \bar{Q}}^{I}\right)=\frac{2}{\pi^{1 / 4}\left(\beta_{q \bar{Q}}^{V, P}\right)^{3 / 2}} \exp \left[-\frac{\mathrm{k}^{2}}{2\left(\beta_{q \bar{Q}}^{I}\right)^{2}}\right]
$$

from integral representation and current quark masses we get the following parameters of the model under consideration:

$$
\begin{gathered}
m_{u}=(219.48 \pm 9.69) \mathrm{MeV}, m_{d}=(221.97 \pm 9.69) \mathrm{MeV}, m_{s}=(416.95 \pm 61.22) \mathrm{MeV} \\
\beta_{u \bar{d}}^{P}=(367.93 \pm 25.10) \mathrm{MeV}, \quad \beta_{u \bar{d}}^{V}=(311.95 \pm 2.14) \mathrm{MeV} \\
\beta_{u \bar{s}}^{P}=(375.53 \pm 19.66) \mathrm{MeV}, \quad \beta_{u \bar{s}}^{V}=(313.62 \pm 24.22) \mathrm{MeV}
\end{gathered}
$$

(analysis of the results was carried out in [5]). For further calculations, using the weak isotopic symmetry violation, we assume, that

$$
\beta_{u \bar{u}}^{V}=\beta_{u \bar{d}}^{V}-\Delta \beta_{u \bar{d}}, \quad \beta_{d \bar{d}}^{V}=\beta_{u \bar{d}}^{V}+\Delta \beta_{u \bar{d}}, \quad \beta_{d \bar{s}}^{V}=\beta_{u \bar{s}}^{V}+\Delta \beta_{u \bar{d}}, \quad \beta_{d \bar{s}}^{P}=\beta_{u \bar{s}}^{P}+\Delta \beta_{u \bar{d}},
$$

where $\Delta \beta_{u \bar{d}} \simeq m_{d}-m_{u}=(2.5 \pm 0.2) \mathrm{MeV}$. 


\section{Radiative decay constant in PiQM}

Parameterization of the matrix element for the vector (pseudoscalar) meson $V(P)$ transition into a pseudoscalar (vector) meson $P(V)$ with 4-momenta $Q=\left\{Q_{0}, \mathbf{Q}\right\}\left(Q^{2}=M^{2}\right)$ and $Q^{\prime}=$ $\left\{Q_{0}^{\prime}, \mathbf{Q}^{\prime}\right\}\left(Q^{\prime 2}=M^{\prime 2}\right)$ by emitting a virtual $\gamma^{*}$ is given by [6]:

$$
\left\langle\mathbf{Q}^{\prime}, M^{\prime}\left|\hat{J}_{h}^{\mu}(0)\right| \mathbf{Q}, M\right\rangle=i \sqrt{4 \pi \alpha} g_{V P \gamma^{*}}\left(q^{2}\right) \frac{\epsilon^{\mu \nu \rho \sigma} \varepsilon_{v}\left(\lambda_{V}\right) Q_{\rho} Q_{\sigma}}{(2 \pi)^{3} \sqrt{4 Q_{0} Q_{0}^{\prime}}},
$$

where $\varepsilon_{v}\left(\lambda_{V}\right)$ is a polarization vector of the meson $V, \alpha=e^{2} / 4 \pi-$ a fine structure constant and $q=Q-Q^{\prime}$.

In the proposed approach the decay constant of $V(P) \rightarrow P(V) \gamma^{*}$ from (5) is defined by the following:

$$
g_{V P \gamma^{*}}\left(q^{2}\right)=(2 \pi)^{3}\left\langle\mathbf{Q}^{\prime}, M^{\prime}\left|\frac{\sqrt{4 V_{0}^{\prime} V_{0}}}{\sqrt{M_{0}\left(\mathrm{k}^{\prime}\right) M_{0}(\mathrm{k})}} \frac{\left(\hat{J}_{h}(0) \cdot K^{*}\left(\lambda_{V}\right)\right)}{\left(K\left(\lambda_{V}\right) \cdot K^{*}\left(\lambda_{V}\right)\right)}\right| \mathbf{Q}, M\right\rangle,
$$

where $K^{\mu}\left(\lambda_{V}\right)=i \epsilon^{\mu \nu \rho \sigma} \varepsilon_{v}\left(\lambda_{V}\right) V_{\rho} V_{\sigma}^{\prime}$. Assuming, that the studied decay caused by interaction of constituent quarks, which are included in the meson $V(P)$, and virtual $\gamma$-quantum with vertex

$$
\Gamma_{q, \bar{Q}}^{\mu}=F_{1}\left(q^{2}\right) \gamma^{\mu}+\frac{1}{2 m_{q, \bar{Q}}} F_{2}\left(q^{2}\right) \sigma^{\mu v} q_{v}
$$

where

$$
F_{1}(0)+F_{2}(0)=\mu_{q, \bar{Q}}, \quad \mu_{q, \bar{Q}}=\frac{e_{q, \bar{Q}}}{2 m_{q, \bar{Q}}}\left(1+\kappa_{q, \bar{Q}}\right),
$$

in the generalized Breit system $\left(\mathbf{V}_{Q}+\mathbf{V}_{Q^{\prime}}=0\right)$ with limiting $q^{2} \rightarrow 0$ one can obtain the integral representation of radiative decay constant for $V \rightarrow P \gamma$ decay [4]:

$$
\begin{gathered}
g_{V P \gamma}=\int_{0}^{\infty} \mathrm{dk} \mathrm{k}{ }^{2} \Phi\left(\mathrm{k}, \beta_{q \bar{Q}}^{V}\right) \Phi^{*}\left(\mathrm{k}, \beta_{q \bar{Q}}^{P}\right)\left(e_{q} f_{1}\left(\mathrm{k}, m_{q}, m_{\bar{Q}}\right)+\right. \\
\left.+\frac{e_{q} \kappa_{q}}{2 m_{q}} f_{2}\left(\mathrm{k}, m_{q}, m_{\bar{Q}}\right)-e_{\bar{Q}} f_{1}\left(\mathrm{k}, m_{\bar{Q}}, m_{q}\right)-\frac{e_{\bar{Q}} \kappa_{\bar{Q}}}{2 m_{\bar{Q}}} f_{2}\left(\mathrm{k}, m_{\bar{Q}}, m_{q}\right)\right),
\end{gathered}
$$

where

$$
\begin{array}{r}
f_{1}\left(\mathrm{k}, m_{q}, m_{\bar{Q}}\right)=\frac{1}{3 \omega_{m_{q}}(\mathrm{k})}\left(\frac{m_{q}+m_{\bar{Q}}}{M_{0}(\mathrm{k})}+\frac{m_{q}}{\omega_{m_{q}}(\mathrm{k})}+1\right) \\
f_{2}\left(\mathrm{k}, m_{q}, m_{\bar{Q}}\right)=-\frac{2}{3}\left(\frac{m_{q}^{2}+\omega_{m_{q}}(\mathrm{k})\left(m_{q}+\omega_{m_{q}}(\mathrm{k})\right)}{\omega_{m_{q}}^{2}(\mathrm{k})}\right)
\end{array}
$$

and

$$
M_{0}(\mathrm{k})=\omega_{m_{q}}(\mathrm{k})+\omega_{m_{\bar{Q}}}(\mathrm{k}), \omega_{m}(\mathrm{k})=\sqrt{\mathrm{k}^{2}+m^{2}}, \mathrm{k}=|\mathbf{k}| .
$$

Fixing the value of quark ( $u, d$ and $s$ ) magnetic moments will be carried out from the requirement coincidence of theoretical calculations with experimental data [1]. Using $\rho^{+}, K^{*+}$ and $K^{* 0}$ decay values from (9) one can obtain the following values: $\kappa_{u}=(-0.123 \pm 0.084), \kappa_{d}=$ $(-0.088 \pm 0.015)$ and $\kappa_{s}=(-0.198 \pm 0.011)$ (we use natural quark units). The analysis has shown that values of anomalous magnetic moments in our work are in good agreement with other models and assumptions: in [7] the used values $\tilde{\kappa}_{u}=-0.064, \tilde{\kappa}_{d}=0.017$; comparing to our results one can get $\tilde{\kappa}_{u}=\kappa_{u} / e_{u}=-0.082, \tilde{\kappa}_{d}=\kappa_{d} / e_{d}=0.029$. 


\section{Mixing scheme of mesons}

Due to various mixing effects the mesons are usually a linear combination of state vectors (1), which differ from each other by their quark composition. So in this section we briefly discuss the mixing scheme scenarios for the unflavored pseudoscalar and vector mesons.

In physical applications mixing schemes with bases [8]

$$
\left\{\begin{array} { l } 
{ \psi _ { 1 } = ( 1 / \sqrt { 2 } ) | u \overline { u } - d \overline { d } \rangle , } \\
{ \psi _ { q } = ( 1 / \sqrt { 2 } ) | u \overline { u } + d \overline { d } \rangle , } \\
{ \psi _ { s } = | s \overline { s } \rangle }
\end{array} \quad \text { and } \quad \left\{\begin{array}{l}
\psi_{1}=(1 / \sqrt{2})|u \bar{u}-d \bar{d}\rangle, \\
\psi_{8}=(1 / \sqrt{6})|u \bar{u}+d \bar{d}-2 s \bar{s}\rangle, \\
\psi_{0}=(1 / \sqrt{3})|u \bar{u}+d \bar{d}+s \bar{s}\rangle .
\end{array}\right.\right.
$$

are widely used. By means of (12) the physical states of vector mesons are defined by

$$
\left(\begin{array}{c}
|\phi\rangle \\
|\omega\rangle \\
\left|\rho^{0}\right\rangle
\end{array}\right)=U\left(\phi_{V}, \phi_{\rho \phi}, \phi_{\rho \omega}\right)\left(\begin{array}{c}
\psi_{q} \\
\psi_{s} \\
\psi_{1}
\end{array}\right)=U\left(\theta_{V}, \theta_{\rho \phi}, \theta_{\rho \omega}\right)\left(\begin{array}{l}
\psi_{8} \\
\psi_{0} \\
\psi_{1}
\end{array}\right)
$$

where $U(\alpha, \beta, \gamma)$ - rotation matrix.

It is known, that $\phi_{\rho \omega}$-mixing angle is due to QCD processes, which is the complex number [9], whose numerical value is obtained with a large error, so we assume that $\phi_{\rho \omega}=0 ; \rho-\phi-$ mixing can be also neglected. In the final analysis we will use only one $\phi_{V}$-angle for the vector meson sector, for which it follows from (13) that:

$$
\left\{\begin{array}{l}
|\phi\rangle=\cos \phi_{V} \psi_{q}-\sin \phi_{V} \psi_{s} \\
|\omega\rangle=\sin \phi_{V} \psi_{q}+\cos \phi_{V} \psi_{s} \\
\left|\rho^{0}\right\rangle=\psi_{1}
\end{array}\right.
$$

where $\phi_{V}$-angle, related with the $\theta_{V}$-angle [8] by

$$
\theta_{V}=\phi_{V}-\arctan \sqrt{2}
$$

For the pseudoscalar sector, respectively,

$$
\left(\begin{array}{c}
|\eta\rangle \\
\left|\eta^{\prime}\right\rangle \\
\left|\pi^{0}\right\rangle
\end{array}\right)=U\left(\phi_{P}, \phi_{\pi \eta}, \phi_{\pi \eta^{\prime}}\right)\left(\begin{array}{c}
\psi_{q} \\
\psi_{s} \\
\psi_{1}
\end{array}\right)
$$

where mixing effects for $\pi^{0}-\eta$ and $\pi^{0}-\eta^{\prime}$ are extremely small, so a naive mixing scheme form (16) leads to the following:

$$
\left\{\begin{array}{l}
|\eta\rangle=\cos \phi_{P} \psi_{q}-\sin \phi_{P} \psi_{s} \\
\left|\eta^{\prime}\right\rangle=\sin \phi_{P} \psi_{q}+\cos \phi_{P} \psi_{s} \\
\left|\pi^{0}\right\rangle=\psi_{1}
\end{array}\right.
$$

However, the decay $\eta-\eta^{\prime}$ analysis has shown, that scheme (17) with one mixing angle is not enough to describe the experimental data, therefore the number of independent mixing parameters $\eta-\eta^{\prime}$ was increased due to the gluonium content [2]. As a result the physical states for pseudoscalar mesons could be written as [10]:

$$
\left(\begin{array}{c}
|\eta\rangle \\
\left|\eta^{\prime}\right\rangle \\
|G\rangle
\end{array}\right)=U\left(\phi_{P}, \alpha_{G}, \phi_{G}\right)\left(\begin{array}{c}
\psi_{q} \\
\psi_{s} \\
\psi_{G}
\end{array}\right)
$$


or, using an explicit form of the $U\left(\phi_{P}, \alpha_{G}, \phi_{G}\right)$-matrix

$$
\left\{\begin{array}{l}
|\eta\rangle=X_{\eta} \psi_{q}+Y_{\eta} \psi_{s}+Z_{\eta} \psi_{G}, \\
\left|\eta^{\prime}\right\rangle=X_{\eta^{\prime}} \psi_{q}+Y_{\eta^{\prime}} \psi_{s}+Z_{\eta^{\prime}} \psi_{G}, \\
|G\rangle=X_{G} \psi_{q}+Y_{G} \psi_{s}+Z_{G} \psi_{G},
\end{array}\right.
$$

where $|G\rangle$ is a gluonium content and

$$
X_{\eta}=\cos \phi_{P} \cos \alpha_{G}, \quad Y_{\eta}=-\sin \phi_{P} \cos \alpha_{G}, \quad Z_{\eta}=-\sin \alpha_{G},
$$

$X_{\eta^{\prime}}=\cos \phi_{P} \sin \alpha_{G} \sin \phi_{G}+\sin \phi_{P} \cos \phi_{G}, \quad Y_{\eta^{\prime}}=\cos \phi_{P} \cos \phi_{G}-\sin \phi_{P} \sin \alpha_{G} \sin \phi_{G}$,

$$
Z_{\eta^{\prime}}=\cos \alpha_{G} \sin \phi_{G}
$$

Using these mixing schemes and taking into account the fact, that state vectors orthogonal for different quark flavours for various $V(P) \rightarrow P(V) \gamma$ decays, the decay constant in PiQM can be represented as in Tab. 1.

Table 1. Decay analysis in PiQM

\begin{tabular}{c|c}
\hline Radiative decay & Decay constant representation in PiQM \\
\hline$\rho^{0} \rightarrow \pi^{0} \gamma$ & $e_{u} I(u \bar{u})+e_{d} I(d \bar{d})$ \\
$\rho^{0} \rightarrow \eta \gamma$ & $X_{\eta}\left(e_{u} I(u \bar{u})-e_{d} I(d \bar{d})\right)$ \\
$\phi \rightarrow \pi^{0} \gamma$ & $\cos \phi_{V}\left(e_{u} I(u \bar{u})-e_{d} I(d \bar{d})\right)$ \\
$\phi \rightarrow \eta \gamma$ & $\cos \phi_{V} X_{\eta}\left(e_{u} I(u \bar{u})+e_{d} I(d \bar{d})\right)-2 e_{s} I(s \bar{s}) \sin \phi_{V} Y_{\eta}$ \\
$\phi \rightarrow \eta \gamma$ & $\cos \phi_{V} X_{\eta^{\prime}}\left(e_{u} I(u \bar{u})+e_{d} I(d \bar{d})\right)-2 e_{s} I(s \bar{s}) \sin \phi_{V} Y_{\eta^{\prime}}$ \\
$\omega \rightarrow \pi^{0} \gamma$ & $\sin \phi_{V}\left(e_{u} I(u \bar{u})-e_{d} I(d \bar{d})\right)$ \\
$\omega \rightarrow \eta \gamma$ & $\sin \phi_{V} X_{\eta}\left(e_{u} I(u \bar{u})+e_{d} I(d \bar{d})\right)+2 e_{s} I(s \bar{s}) \cos \phi_{V} Y_{\eta}$ \\
$\eta^{\prime} \rightarrow \rho^{0} \gamma$ & $X_{\eta^{\prime}}\left(e_{u} I(u \bar{u})-e_{d} I(d \bar{d})\right)$ \\
$\eta^{\prime} \rightarrow \omega \gamma$ & $X_{\eta^{\prime}} \sin \phi_{V}\left(e_{u} I(u \bar{u})+e_{d} I(d \bar{d})\right)+2 e_{s} I(s \bar{s}) \cos \phi_{V} Y_{\eta^{\prime}}$ \\
\hline
\end{tabular}

Note, that in Tab. 1 we have used an abbreviation

$$
I(q \bar{q})=\int_{0}^{\infty} \mathrm{dk} \mathrm{k}^{2} \Phi\left(\mathrm{k}, \beta_{q \bar{q}}^{V}\right) \Phi^{*}\left(\mathrm{k}, \beta_{q \bar{q}}^{P}\right)\left(\frac{\left(\omega_{m_{q}}(\mathrm{k})+2 m_{q}\right)}{3 \omega_{m_{q}}^{2}(\mathrm{k})}+\kappa_{q}\left(-\frac{1}{3} \frac{m_{q}^{2}+\omega_{m_{q}}(\mathrm{k})\left(m_{q}+\omega_{m_{q}}(\mathrm{k})\right)}{m_{q} \omega_{m_{q}}^{2}(\mathrm{k})}\right)\right),
$$

which was obtained from (9-11) taking into account $m_{q}=m_{\bar{Q}}$ and $\left|e_{q}\right|=\left|e_{\bar{Q}}\right|$.

\section{Numerical results and discussion}

Using basic parameters of the model (see section 1) and relation (21) one can obtain the following values for mixing angles and $\beta_{q \bar{q}}^{P}$-parameters from the condition of compliance theoretical calculations with experimental data (see Tab. 1):

$$
\begin{gathered}
\beta_{u \bar{u}}^{P}=(280.60 \pm 25.07) \mathrm{MeV}, \beta_{d \bar{d}}^{P}=(277.95 \pm 25.07) \mathrm{MeV}, \beta_{s \bar{s}}^{P}=(494.54 \pm 19.66) \mathrm{MeV}, \\
\theta_{P}=(-9.1 \pm 2.4)^{\circ}, \alpha_{G}=(9.5 \pm 3.4)^{\circ}, \phi_{G}=(-28.8 \pm 3.4)^{\circ} .
\end{gathered}
$$

Note, that for calculations we have used the mixing angle value $\theta_{V}=(31.92 \pm 0.2)^{\circ}[11]$, which for $\omega$-meson decay into $\ell^{+} \ell^{-}$-pair leads to the following:

$$
\beta_{s \bar{s}}^{V}=(336.56 \pm 1.38) \mathrm{MeV} .
$$

Using the obtained model parameters and quark magnetic moments (see sec.1 and 2) we compare the experimental data with model calculations in Tab. 2: 
Table 2. Comparing experimental $\Gamma^{(\text {exp. })}$ and theoretical values $\Gamma^{(t h .)}$ for $V(P) \rightarrow P(V) \gamma$ decay taking into account anomalous quark magnetic moments

\begin{tabular}{c|c|c}
\hline Radiative decay & $\Gamma^{(\text {exp.) }}, \mathrm{keV}$ & $\Gamma^{(t h .)}, \mathrm{keV}$ \\
\hline$\omega \rightarrow \pi^{0} \gamma$ & $687 \pm 19[3]$ & $704 \pm 13$ \\
& $714 \pm 20[1]$ & \\
\hline$\omega \rightarrow \eta \gamma$ & $5.8 \pm 1.1[1]$ & $6.8 \pm 2.3$ \\
\hline$\phi \rightarrow \pi^{0} \gamma$ & $5.5 \pm 0.2[1]$ & $5.6 \pm 2.1$ \\
\hline$\phi \rightarrow \eta \gamma$ & $55.4 \pm 1.2[1]$ & $55.7 \pm 2.5$ \\
\hline$\phi \rightarrow \eta \gamma$ & $0.27 \pm 0.01[1]$ & $0.27 \pm 0.14$ \\
\hline$\rho^{0} \rightarrow \pi^{0} \gamma$ & $77 \pm 28[12]$ & $83 \pm 4$ \\
\hline$\rho^{0} \rightarrow \eta \gamma$ & $44.7 \pm 3.2[1]$ & $44.8 \pm 3.5$ \\
\hline$\eta^{\prime} \rightarrow \rho^{0} \gamma$ & $56.7 \pm 2.7[1]$ & $56.9 \pm 2.3$ \\
\hline$\eta^{\prime} \rightarrow \omega \gamma$ & $5.1 \pm 0.4[1]$ & $5.1 \pm 0.8$ \\
\hline \multicolumn{2}{|c}{}
\end{tabular}

\section{Conclusion}

The work is dedicated to the calculations of the integral representation radiative decay constants of pseudoscalar and vector mesons. In the course of the work the authors basing on the previously obtained results of quark masses and $\beta_{q \bar{Q}}$-parameters of wave functions, estimated the values of anomalous quark magnetic moments and mixing angles for pseudoscalar and vector mesons with gluonium content. These values have been compared and do not contradict other magnitudes in different models and approaches.

It should be noted, that the results of the model, based on the point-form of PiQM, are self-consistent, what makes it possible to use the proposed model to research the behaviour of $g_{V P \gamma^{*}}$ for different square transfer momentum $t=q^{2}$.

Acknowledgments. This work was supported by grant F17D-001 of the Belarusian Republican Foundation for Fundamental Research (Minsk, Republic of Belarus).

\section{References}

[1] M. Tanabashi et al., Phys. Rev. D 98, 030001 (2018)

[2] F. Ambrosino et al. [KLOE Collaboration], Phys. Lett.B 648, 267-273 (2007)

[3] F. Ambrosino et al. [KLOE Collaboration], JHEP 7, 105 (2009)

[4] V.V Andreev, V.Yu. Haurysh, J. Phys.: Conf. Ser. 678, 012041 (2016)

[5] V.V Andreev, V.Yu. Haurysh, J. Phys.: Conf. Ser. 938, 012030 (2017)

[6] W. Jaus, Phys. Rev. D 44, 2851-2859 (1991)

[7] R. Petronzio and S. Simula and G. Ricco, Phys. Rev. D 67, 094004 (2003)

[8] A. Bramon, R. Escribano, M. D. Scadron, Phys. Lett. B 503, 271-276 (2001)

[9] R. P. Feynman, Photon-hadron interactions (W.A. Benjamin, USA, 1972) 300

[10] C. E. Thomas, JHEP, 10, 26 (2007)

[11] G. Amelino-Camelia et al. [KLOE Collaboration], Eur. Phys. J. 68, 619-681 (2010)

[12] M. N. Achasov et al. [SND Collaboration], Phys. Rev. D 93, 092001 (2016) 